\title{
From constant to non-degenerately vanishing magnetic fields in superconductivity
}

\author{
Bernard Helffer ${ }^{\mathrm{a}, \mathrm{b}}$, Ayman Kachmar ${ }^{\mathrm{c}, *}$ \\ a Laboratoire de Mathématiques, Université de Paris-Sud 11, Bât 425, 91405 Orsay, France \\ ${ }^{\mathrm{b}}$ Laboratoire Jean Leray (Université de Nantes), France \\ ${ }^{\mathrm{c}}$ Department of Mathematics, Lebanese University, Hadat, Lebanon
}

Received 2 April 2015; accepted 23 December 2015

Available online 15 January 2016

\begin{abstract}
We explore the relationship between two reference functions arising in the analysis of the Ginzburg-Landau functional. The first function describes the distribution of superconductivity in a type II superconductor subjected to a constant magnetic field. The second function describes the distribution of superconductivity in a type II superconductor submitted to a variable magnetic field that vanishes non-degenerately along a smooth curve.
\end{abstract}

(c) 2016 Elsevier Masson SAS. All rights reserved.

Keywords: Ginzburg-Landau functional; Non-degenerately vanishing magnetic fields; Energy asymptotics

\section{Introduction}

The Ginzburg-Landau functional is a celebrated phenomenological model that describes the response of a superconductor to a magnetic field [8]. In non-dimensional units, the functional is defined as follows,

$$
\mathcal{E}(\psi, \mathbf{A})=\int_{\Omega}\left(|(\nabla-i \mathbf{A}) \psi|^{2}-\kappa^{2}|\psi|^{2}+\frac{\kappa^{2}}{2}|\psi|^{4}+\left|\operatorname{curl} \mathbf{A}-h_{\mathrm{ex}} B_{0}\right|^{2}\right) d x,
$$

where:

- $\Omega \subset \mathbb{R}^{2}$ is an open, bounded and simply connected set with a smooth boundary; $\Omega$ is the cross section of a cylindrical superconducting sample placed vertically;

- $(\psi, \mathbf{A}) \in H^{1}(\Omega ; \mathbb{C}) \times H^{1}\left(\Omega ; \mathbb{R}^{2}\right)$ describe the state of superconductivity as follows: $|\psi|$ measures the density of the superconducting Cooper pairs and curl $\mathbf{A}$ measures the induced magnetic field in the sample;

\footnotetext{
* Corresponding author.

E-mail addresses: bernard.helffer@math.u-psud.fr (B. Helffer), ayman.kashmar@gmail.com (A. Kachmar).
} 
- $\kappa>0$ is the Ginzburg-Landau parameter, a material characteristic of the sample;

- $h_{\mathrm{ex}}>0$ measures the intensity of the applied magnetic field;

- $B_{0}$ is a smooth function defined in $\bar{\Omega}$. The applied magnetic field is $h_{\mathrm{ex}} B_{0} \vec{e}$, where $\vec{e}=(0,0,1)$.

We introduce the ground state energy of the functional in (1.1) as follows,

$$
\mathrm{E}_{\mathrm{gs}}\left(\kappa, h_{\mathrm{ex}} ; B_{0}\right)=\inf \left\{\mathcal{E}(\psi, \mathbf{A}):(\psi, \mathbf{A}) \in H^{1}(\Omega ; \mathbb{C}) \times H^{1}\left(\Omega ; \mathbb{R}^{2}\right)\right\} .
$$

In physical terms, (1.2) describes the energy of a type II superconductor submitted to a possibly non-constant magnetic field of intensity $h_{\mathrm{ex}}\left|B_{0}\right|$.

The behavior of the ground state energy in (1.2) strongly depends on the values of $\kappa$ and $h_{\mathrm{ex}}$. This is the subject of a vast mathematical literature. In the two monographs [6,14], a survey of many important results regarding the behavior of $\mathrm{E}_{\mathrm{gs}}\left(\kappa, h_{\mathrm{ex}} ; B_{0}\right)$ is given. The results are valid when $h_{\mathrm{ex}}=h_{\mathrm{ex}}(\kappa)$ is a function of $\kappa$ and $\kappa \rightarrow+\infty$.

Let us recall two important results regarding the ground state energy in (1.2). The first result is obtained in [15] and says, if $b \in(0,1]$ is a constant, $h_{\mathrm{ex}}=b \kappa^{2}$ and $B_{0}=1$, then

$$
\mathrm{E}_{\mathrm{gs}}\left(\kappa, h_{\mathrm{ex}} ; B_{0}\right)=g(b)|\Omega| \kappa^{2}+o\left(\kappa^{2}\right) \quad(\kappa \rightarrow+\infty),
$$

where $g(b)$ is a constant that will be defined in (1.11) below.

The second result is given in [9] and valid under the following assumption on the function $B_{0}$.

Assumption 1.1. Suppose that $B_{0}: \bar{\Omega} \rightarrow \mathbb{R}$ is a smooth function satisfying

- $\left|B_{0}\right|+\left|\nabla B_{0}\right| \geq c$ in $\bar{\Omega}$, where $c>0$ is a constant;

- $\Gamma=\left\{x \in \bar{\Omega}: B_{0}(x)=0\right\}$ is the union of a finite number of simple smooth curves;

- $\Gamma \cap \partial \Omega$ is a finite set.

Under these assumptions on $B_{0}$, if $b>0$ is a constant and $h_{\mathrm{ex}}=b \kappa^{3}$, then,

$$
\mathrm{E}_{\mathrm{gs}}\left(\kappa, h_{\mathrm{ex}} ; B_{0}\right)=\kappa\left(\int_{\Gamma}\left(b\left|\nabla B_{0}(x)\right|\right)^{1 / 3} E\left(b\left|\nabla B_{0}(x)\right|\right) d s(x)\right)+o(\kappa),
$$

where $E(\cdot)$ is a continuous function that will be defined in (1.18) below, and $d s$ is the arc-length measure in $\Gamma$.

In physical terms, (1.4) describes the energy of a type II superconductor subjected to a variable magnetic field that vanishes along a smooth curve. Such magnetic fields are of special importance in the analysis of the Ginzburg-Landau model in surfaces (see [5]).

Magnetic fields satisfying Assumption 1.1 have an early appearance in the literature, for instance in a paper by Montgomery [10]. Pan and Kwek [13] study the breakdown of superconductivity under the Assumption 1.1. They find a constant $c_{0}>0$ such that, if $h_{\mathrm{ex}}=b \kappa^{3}, b>c_{0}$ and $\kappa$ is sufficiently large, then $\mathrm{E}_{\mathrm{gs}}\left(\kappa, h_{\mathrm{ex}} ; B_{0}\right)=0$. Recently, the results of Pan-Kwek have been improved in [3,11]. The discussion in [9] proves that the formula in (1.4) is consistent with the conclusion in [13] and with Theorem 1.7 in [3].

As proven in [9], the formula in (1.4) continues to hold when $h_{\mathrm{ex}}=b \kappa^{3}$ and $b=b(\kappa)$ satisfies, ${ }^{1}$

$$
\kappa^{-1 / 2} \ll b(\kappa) \ll 1 \quad(\kappa \rightarrow+\infty) .
$$

When the condition in (1.5) is violated by allowing ${ }^{2}$

$$
\kappa^{-1} \ll b(\kappa) \lesssim \kappa^{-1 / 2} \quad(\kappa \rightarrow+\infty)
$$

then the formula in (1.4) is replaced with (see [9]),

$$
\mathrm{E}_{\mathrm{gs}}\left(\kappa, h_{\mathrm{ex}} ; B_{0}\right)=\kappa^{2} \int_{\Omega} g\left(b(\kappa) \kappa\left|B_{0}(x)\right|\right) d x+o\left(b(\kappa)^{-1} \kappa\right) .
$$

\footnotetext{
1 The notation $a(\kappa) \ll b(\kappa)$ means that $a(\kappa)=\delta(\kappa) b(\kappa)$ and $\lim _{\kappa \rightarrow+\infty} \delta(\kappa)=0$.

2 The notation $a(\kappa) \lesssim b(\kappa)$ means that there exists a constant $c>0$ and $\kappa_{0}>0$ such that, for all $\kappa \geq \kappa_{0}, a(\kappa) \leq c b(\kappa)$.
} 
Note that (1.6) is still true for lower values of the external field but with a different expression for the remainder term (see $[1,2])$.

The comparison of the formulas in (1.4) and (1.6) at the border regime ${ }^{3}$

$$
b(\kappa) \approx \kappa^{-1 / 2}
$$

suggests that there might exist a relation between the two reference functions $g(\cdot)$ and $E(\cdot)$. This paper confirms the existence of such a relationship.

The two functions $g(\cdot)$ and $E(\cdot)$ are defined via simplified versions of the functional in (1.1). As we shall see, $g(\cdot)$ will be defined via a constant magnetic field, while, for $E(\cdot)$, this will be via a magnetic field that vanishes along a line.

Let us recall the definition of the function $g(\cdot)$. Consider $b \in(0,+\infty), r>0$, and $Q_{r}=(-r / 2, r / 2) \times$ $(-r / 2, r / 2)$. Define the functional,

$$
F_{b, Q_{r}}(u)=\int_{Q_{r}}\left(b\left|\left(\nabla-i \mathbf{A}_{0}\right) u\right|^{2}-|u|^{2}+\frac{1}{2}|u|^{4}\right) d x, \quad \text { for } u \in H^{1}\left(Q_{r}\right) .
$$

Here, $\mathbf{A}_{0}$ is the magnetic potential,

$$
\mathbf{A}_{0}(x)=\frac{1}{2}\left(-x_{2}, x_{1}\right), \quad \text { for } x=\left(x_{1}, x_{2}\right) \in \mathbb{R}^{2} .
$$

Define the two Dirichlet and Neumann ground state energies,

$$
\begin{aligned}
& e_{D}(b, r)=\inf \left\{F_{b, Q_{r}}(u): u \in H_{0}^{1}\left(Q_{r}\right)\right\}, \\
& e_{N}(b, r)=\inf \left\{F_{b, Q_{r}}(u): u \in H^{1}\left(Q_{r}\right)\right\} .
\end{aligned}
$$

Thanks to $[1,7,15], g(\cdot)$ may be defined as follows,

$$
\forall b>0, \quad g(b)=\lim _{r \rightarrow \infty} \frac{e_{D}(b, r)}{\left|Q_{r}\right|}=\lim _{r \rightarrow \infty} \frac{e_{N}(b, r)}{\left|Q_{r}\right|},
$$

where $\left|Q_{r}\right|$ denotes the area of $Q_{r}\left(\left|Q_{r}\right|=r^{2}\right)$.

Moreover the function $g(\cdot)$ is a non decreasing continuous function such that

$$
g(0)=-\frac{1}{2} \text { and } g(b)=0 \text { when } b \geq 1 .
$$

Now we introduce the function $E(\cdot)$.

Let $L>0, R>0, \mathcal{S}_{R}=(-R / 2, R / 2) \times \mathbb{R}$ and

$$
\mathbf{A}_{\mathrm{van}}(x)=\left(-\frac{x_{2}^{2}}{2}, 0\right), \quad \text { for } x=\left(x_{1}, x_{2}\right) \in \mathbb{R}^{2} .
$$

Notice that $\mathbf{A}_{\mathrm{van}}$ is a magnetic potential generating the magnetic field

$$
B_{\mathrm{van}}(x)=\operatorname{curl} \mathbf{A}_{\mathrm{van}}=x_{2},
$$

which vanishes along the $x_{1}$-axis.

Consider the functional

$$
\mathcal{E}_{L, R}(u)=\int_{\mathcal{S}_{R}}\left(\left|\left(\nabla-i \mathbf{A}_{\mathrm{van}}\right) u\right|^{2}-L^{-2 / 3}|u|^{2}+\frac{L^{-2 / 3}}{2}|u|^{4}\right) d x,
$$

and the ground state energy

$$
\mathfrak{e}_{\mathrm{gs}}(L ; R)=\inf \left\{\mathcal{E}_{L, R}(u): u \in H_{\mathrm{mag}, 0}^{1}\left(\mathcal{S}_{R}\right)\right\},
$$

\footnotetext{
3 The notation $a(\kappa) \approx b(\kappa)$ means that $a(\kappa) \lesssim b(\kappa)$ and $b(\kappa) \lesssim a(\kappa)$.
} 
where

$$
H_{\text {mag }, 0}^{1}\left(\mathcal{S}_{R}\right)=\left\{u \in L^{2}\left(\mathcal{S}_{R}\right):\left(\nabla-i \mathbf{A}_{\mathrm{van}}\right) u \in L^{2}\left(\mathcal{S}_{R}\right) \quad \text { and } \quad u=0 \text { on } \partial \mathcal{S}_{R}\right\} .
$$

Thanks to [9], we may define $E(\cdot)$ as follows,

$$
E(L)=\lim _{R \rightarrow \infty} \frac{\mathfrak{e}_{\mathrm{gs}}(L ; R)}{R} .
$$

In this paper, we obtain a relationship between the functions $E(\cdot)$ and $g(\cdot)$ :

Theorem 1.2. Let $g(\cdot)$ and $E(\cdot)$ be as in (1.11) and (1.18) respectively. It holds,

$$
E(L)=2 L^{-4 / 3} \int_{0}^{1} g(b) d b+o\left(L^{-4 / 3}\right) \quad \text { as } L \rightarrow 0_{+} .
$$

As a consequence of Theorem 1.2 and the co-area formula, we obtain:

Theorem 1.3. Suppose that the function $B_{0}$ satisfies Assumption 1.1 and

$$
\kappa^{-1} \ll b(\kappa) \ll 1 .
$$

Let $g(\cdot)$ and $E(\cdot)$ be the energies introduced in (1.3) and (1.6) respectively. It holds,

$$
\begin{aligned}
\int_{\Omega} & g\left(b(\kappa) \kappa\left|B_{0}(x)\right|\right) d x \\
& \left.=\kappa^{-1} \int_{\Gamma}\left(b(\kappa)\left|\nabla B_{0}(x)\right|\right)^{1 / 3} E\left(b(\kappa)\left|\nabla B_{0}(x)\right|\right) d s(x)+o\left(b(\kappa)^{-1} \kappa^{-1}\right)\right), \quad(\kappa \rightarrow+\infty) .
\end{aligned}
$$

This yields the following improvement of the main result in [9]:

Theorem 1.4. Suppose that Assumption 1.1 holds and

$$
h_{\mathrm{ex}}=b(\kappa) \kappa^{3}, \quad \kappa^{-1} \ll b(\kappa) \lesssim 1 \quad(\kappa \rightarrow+\infty) .
$$

The ground state energy in (1.4) satisfies,

$$
\mathrm{E}_{\mathrm{gs}}\left(\kappa, h_{\mathrm{ex}} ; B_{0}\right)=\kappa \int_{\Gamma}\left(b(\kappa)\left|\nabla B_{0}(x)\right|\right)^{1 / 3} E\left(b(\kappa)\left|\nabla B_{0}(x)\right|\right) d s(x)+o\left(b(\kappa)^{-1} \kappa\right) . \quad(\kappa \rightarrow+\infty) .
$$

The rest of the paper is devoted to the proof of Theorems 1.2 and 1.3. Note that, along the proof of Theorem 1.2, we provide explicit estimates of the remainder terms (see Theorems 3.1 and 4.1).

\section{Preliminaries}

In this section, we collect useful results regarding the two functionals in (1.3) and (1.4).

For the functional in (1.3) and the corresponding ground state energies in (1.9) and (1.10), the following results are given in $[2,7]$ :

\section{Proposition 2.1.}

(1) There exist minimizers of the ground state energies in (1.9) and (1.10).

(2) For all $r>0$ and $b>0$, a minimizer $u_{b, r}$ of (1.9) or (1.10) satisfies

$$
\left|u_{b, r}\right| \leq 1 \quad \text { in } Q_{r} .
$$


(3) For all $r>0$ and $b>0, e_{D}(b, R) \geq e_{N}(b, R)$.

(4) For all $r>0$ and $b \geq 1, e_{D}(b, r)=0$.

(5) There exists a constant $C>0$ such that, for all $b>0$ and $r \geq 1$, then

$$
e_{N}(b, R) \geq e_{D}(b, r)-C r \sqrt{b} .
$$

(6) There exists a constant $C$ such that, for all $r \geq 1$ and $b \in(0,1)$,

$$
g(b) \leq \frac{e_{D}(b, r)}{\left|Q_{r}\right|} \leq g(b)+C \frac{\sqrt{b}}{r} .
$$

Remark 2.2. The estimate in (2.2) continues to hold when $b \geq 1$, since in this case $g(b)=0$ and $e_{D}(b, r)=0$.

Remark 2.3. Let us mention that Inequality (2.1) is proved in [2, Prop. 2.2] for $0<b<1$ and can be easily extended for $b=1$. For $b \geq 1$, we have, $e_{D}(b, R)=0$, and by a simple comparison argument,

$$
e_{N}(b, r) \geq e_{N}(1, r) \geq e_{D}(1, r)-C r=e_{D}(b, r)-C r \geq e_{D}(b, r)-C r \sqrt{b} .
$$

Remark 2.4. We recall the following simple consequence of the assertions (3)-(6) in Proposition 2.1. Knowing that $g(b)=0$ for all $b \geq 1$, we may find a constant $C>0$ such that, for all $b>0$ and $r \geq 1$,

$$
\frac{e_{N}(b, r)}{\left|Q_{r}\right|} \geq g(b)-C \frac{\sqrt{b}}{r} .
$$

The next lemma indicates a regime where the Neumann energy in (1.10) vanishes.

Lemma 2.5. There exists a constant $r_{0}>0$ such that, for all $r \geq r_{0}$ and $b \geq r_{0}$,

$$
e_{N}(b, r)=0 \text {. }
$$

Proof. We have the trivial upper bound, valid for all $b>0$ and $r>0$,

$$
e_{N}(b, r) \leq F_{b, Q_{r}}(0)=0 \text {. }
$$

Now we will prove that $e_{N}(b, r) \geq 0$ for sufficiently large values of $b$ and $r$. Let $u$ be an arbitrary function in $H^{1}\left(Q_{r}\right)$.

We apply a rescaling to obtain,

$$
\int_{Q_{r}}\left|\left(\nabla-i \mathbf{A}_{0}\right) u\right|^{2} d x=r^{4} \int_{Q_{1}}\left|\left(r^{-2} \nabla-i \mathbf{A}_{0}\right) v\right|^{2} d y,
$$

where

$$
v(y)=u(r y) .
$$

For every $h>0$, we introduce the following ground state eigenvalue,

$$
\mu_{1}(h)=\inf _{\substack{v \in H^{1}\left(Q_{1}\right) \\ v \neq 0}} \frac{\int_{Q_{1}}\left|\left(h \nabla-i \mathbf{A}_{0}\right) v\right|^{2} d y}{\int_{Q_{1}}|v|^{2} d y} .
$$

It is a known fact that (see $[4,12,6])$,

$$
\lim _{h \rightarrow 0_{+}} \frac{\mu_{1}(h)}{h}=\Theta_{1},
$$

where $\Theta_{1} \in(0,1)$ is a universal constant. 
In that way, we get a constant $r_{1}>0$ such that, for all $r \geq r_{1}$, we infer from (2.3),

$$
\int_{Q_{r}}\left|\left(\nabla-i \mathbf{A}_{0}\right) u\right|^{2} d x \geq \frac{\Theta_{1}}{2} \int_{Q_{1}}|v(y)|^{2} r^{2} d y=\frac{\Theta_{1}}{2} \int_{Q_{r}}|u(x)|^{2} d x .
$$

We insert this into the expression of $F_{b, Q_{r}}(u)$ to get, for all $r \geq r_{1}$ and $b>0$,

$$
F_{b, Q_{r}}(u) \geq \int_{Q_{r}}\left(b \frac{\Theta_{1}}{2}-1\right)|u|^{2} d x .
$$

Let $r_{0}=\max \left(r_{1}, 2 \Theta_{1}^{-1}\right)$. Clearly, for all $r \geq r_{0}, b \geq r_{0}$ and $u \in H^{1}\left(Q_{r}\right), F_{b, Q_{r}}(u) \geq 0$. Consequently, $e_{N}(b, r) \geq 0$.

The functional in (1.4) is studied in [9]. In particular, the following results were obtained:

\section{Proposition 2.6.}

(1) For all $L>0$ and $R>0$, there exists a minimizer $\varphi_{L, R}$ of (1.16).

(2) The function $\varphi_{L, R}$ satisfies

$$
\left|\varphi_{L, R}\right| \leq 1 \quad \text { in } \mathcal{S}_{R}
$$

(3) There exists a constant $C>0$ such that, for all $L>0$ and $R>0$,

$$
\int_{\mathcal{S}_{R}}\left|\varphi_{L, R}(x)\right|^{2} d x \leq C L^{-2 / 3} R .
$$

(4) For all $L>0$ and $R>0$,

$$
E(L) \leq \frac{\mathfrak{e}_{\mathrm{gs}}(L ; R)}{R} .
$$

(5) There exists a constant $C>0$ such that, for all $L>0$ and $R \geq 4$,

$$
\frac{\mathfrak{e}_{\mathrm{gs}}(L ; R)}{R} \leq E(L)+C\left(1+L^{-2 / 3}\right) R^{-2 / 3} .
$$

\section{Proof of Theorem 1.2: lower bound}

The aim of this section is to prove the lower bound in Theorem 1.2. Note that the lower bound below is with a better remainder term.

Theorem 3.1. There exist two constants $L_{0}>0$ and $C>0$ such that, for all $L \in\left(0, L_{0}\right)$,

$$
E(L) \geq 2 L^{-4 / 3} \int_{0}^{1} g(b) d b-C L^{-1},
$$

where $E(\cdot)$ and $g(\cdot)$ are the energies introduced in (1.18) and (1.11) respectively.

The proof of Theorem 3.1 relies on the following lemma:

Lemma 3.2. Let $M>0$. There exist two constants $C>0$ and $A_{0} \geq 4$ such that, if

$$
\begin{aligned}
& A \geq A_{0}, \quad R \geq 1, \quad 0<L \leq A^{-3 / 2}, \quad u \in H^{1}\left(\mathcal{S}_{R}\right), \\
& \|u\|_{\infty} \leq 1 \quad \text { and } \int_{\mathcal{S}_{R}}|u|^{2} d x \leq M L^{-2 / 3} R,
\end{aligned}
$$


then

$$
\int_{\mathcal{S}_{R} \cap\left\{\left|x_{2}\right| \geq A\right\}}\left(\left|\left(\nabla-i \mathbf{A}_{\mathrm{van}}\right) u\right|^{2}-L^{-2 / 3}|u|^{2}+\frac{L^{-2 / 3}}{2}|u|^{4}\right) d x \geq 2 R L^{-4 / 3} \int_{0}^{1} g(b) d b-C R L^{-1} .
$$

Proof. Let $L \in(0,1), A>0$ and $R$ and $u$ satisfy the assumptions in Lemma 3.2. If $\mathcal{D} \subset \mathcal{S}_{R}$, then we use the notation

$$
\mathcal{E}(u ; \mathcal{D})=\int_{\mathcal{D}}\left(\left|\left(\nabla-i \mathbf{A}_{\mathrm{van}}\right) u\right|^{2}-L^{-2 / 3}|u|^{2}+\frac{1}{2} L^{-2 / 3}|u|^{4}\right) d x .
$$

We will prove that,

$$
\mathcal{E}\left(u ; \mathcal{S}_{R} \cap\left\{x_{2} \geq A\right\}\right) \geq R L^{-4 / 3} \int_{0}^{1} g(b) d b-C R L^{-1},
$$

and

$$
\mathcal{E}\left(u ; \mathcal{S}_{R} \cap\left\{x_{2} \leq-A\right\}\right) \geq R L^{-4 / 3} \int_{0}^{1} g(b) d b-C R L^{-1},
$$

for some constant $C$ independent of $L, R, A, L$ and $u$.

We will write the detailed proof of (3.2). The proof of (3.3) is identical.

Let $r_{0}$ be the universal constant introduced in Lemma 2.5. We define $b_{0}=2 \max \left(1, r_{0}^{2}\right)$. Thanks to Lemma 2.5, we have,

$$
\forall b \geq \frac{b_{0}}{2}, \quad \forall r \geq \sqrt{b_{0}}, \quad e_{N}(b, r)=0,
$$

where $e_{N}$ is the Neumann ground state energy introduced in (1.10).

We define the constant $A_{0}=4 \sqrt{b_{0}}$. We introduce $n \in \mathbb{N}$ and

$$
\ell=n^{-1} R \text {. }
$$

We will fix a choice of $n$ later at the end of this proof such that (for all $A \geq A_{0}$ ),

$$
R<n \leq \frac{\sqrt{A} R}{2 \sqrt{b_{0}}}
$$

which ensures that $0<\ell<1$, some $n$ always exists, and

$$
\sqrt{A} \ell \geq 2 \sqrt{b_{0}} .
$$

Let $\left(Q_{\ell, j}\right)_{j \in \mathcal{J}}$ be the lattice of squares generated by

$$
Q_{\ell}=(-R / 2,-R / 2+\ell) \times(A, A+\ell),
$$

and covering $\mathbb{R}^{2} \backslash\left\{x_{2} \leq A\right\}$.

For every $j \in \mathcal{J}$, let $c_{j}=\left(c_{j, 1}, c_{j, 2}\right) \in \mathbb{R}^{2}$ be the center of the square $Q_{\ell, j}$, i.e.

$$
Q_{\ell, j}=\left(-\ell / 2+c_{j, 1}, \ell / 2+c_{j, 1}\right) \times\left(-\ell / 2+c_{j, 2}, \ell / 2+c_{j, 2}\right) .
$$

Let $\mathbf{A}_{0}$ be the magnetic potential in (1.8), $j \in \mathcal{J}, a_{j}=\left(a_{j, 1}, a_{j, 2}\right) \in \overline{Q_{\ell, j}}$ be an arbitrary point and

$$
\mathbf{F}_{j}\left(x_{1}, x_{2}\right)=\left(-\frac{1}{3}\left(x_{2}-a_{j, 2}\right)^{2}, \frac{1}{3}\left(x_{2}-a_{j, 2}\right)\left(x_{1}-a_{j, 1}\right)\right) .
$$

Note that, for the sake of simplicity, we omitted the reference to $\ell$ in the notion of $c_{j}, a_{j}$ and $\mathbf{F}_{j}$. It is easy to check that 


$$
\operatorname{curl} \mathbf{A}_{\mathrm{van}}=\operatorname{curl}\left(a_{j, 2} \mathbf{A}_{0}+\mathbf{F}_{j}\right) \quad \text { in } \quad Q_{\ell, j} .
$$

Since the square $Q_{\ell, j}$ is a simply connected domain in $\mathbb{R}^{2}$, then there exists a real-valued smooth function $f_{j}$ defined in $Q_{\ell, j}$ such that

$$
\mathbf{A}_{\mathrm{van}}=a_{j, 2} \mathbf{A}_{0}+\mathbf{F}_{j}-\nabla f_{j} \quad \text { in } \quad Q_{\ell, j} .
$$

Let us define the smooth function

$$
\phi_{j}(x)=f_{j}(x)-a_{j, 2} \mathbf{A}_{0}\left(c_{j}\right) \cdot x \quad\left(x \in Q_{\ell, j}\right) .
$$

Now, we have,

$$
\mathbf{A}_{\mathrm{van}}(x)=a_{j, 2} \mathbf{A}_{0}\left(x-c_{j}\right)+\mathbf{F}_{j}(x)-\nabla \phi_{j}(x) \text { in } Q_{\ell, j} .
$$

Thanks to the definition of $\mathbf{F}_{j}$, we have,

$$
\left|\mathbf{F}_{j}(x)\right| \leq \ell^{2} \quad \text { in } \quad Q_{\ell, j} .
$$

Now, we write the obvious decomposition formula,

$$
\mathcal{E}\left(u ; \mathcal{S}_{R} \cap\left\{x_{2} \geq A\right\}\right)=\sum_{j \in \mathcal{J}} \mathcal{E}\left(u ; Q_{\ell, j}\right) .
$$

We write a lower bound for $\mathcal{E}\left(u ; Q_{\ell, j}\right)$ when $j \in \mathcal{J}$. Recall that, by assumption, for all $j \in \mathcal{J}, Q_{\ell, j} \subset\left\{x_{2} \geq A\right\}$. Let $0<\eta<\frac{1}{2}$. Thanks to (3.7), we may write,

$$
\begin{aligned}
\mathcal{E}\left(u ; Q_{\ell, j}\right)= & \int_{Q_{\ell, j}}\left(\left|\left(\nabla-i\left(\mathbf{A}_{\mathrm{van}}+\nabla \phi_{j}\right)\right) e^{i \phi_{j}} u\right|^{2}-L^{-2 / 3}\left|e^{i \phi_{j}} u\right|^{2}+\frac{L^{-2 / 3}}{2}\left|e^{i \phi_{j}} u\right|^{4}\right) d x \\
\geq & \int_{Q_{\ell, j}}\left((1-\eta)\left|\left(\nabla-i a_{j, 2} \mathbf{A}_{0}\left(x-c_{j}\right)\right) e^{i \phi_{j}} u\right|^{2}-L^{-2 / 3}\left|e^{i \phi_{j}} u\right|^{2}+\frac{L^{-2 / 3}}{2}\left|e^{i \phi_{j}} u\right|^{4}\right) d x \\
& -4 \eta^{-1} \int_{Q_{\ell, j}}\left|\mathbf{F}_{j}(x)\right|^{2}|u|^{2} d x .
\end{aligned}
$$

Using the bound in (3.8), we get further,

$$
\begin{aligned}
\mathcal{E}\left(u ; Q_{\ell, j}\right) \geq \int_{Q_{\ell, j}}( & (1-\eta)\left|\left(\nabla-i a_{j, 2} \mathbf{A}_{0}\left(x-c_{j}\right)\right) e^{i \phi_{j}} u\right|^{2} \\
& \left.\quad-L^{-2 / 3}\left|e^{i \phi_{j}} u\right|^{2}+\frac{L^{-2 / 3}}{4}\left|e^{i \phi_{j}} u\right|^{2}\right) d x-C \eta^{-1} \ell^{4} \int_{Q_{\ell, j}}|u|^{2} d x .
\end{aligned}
$$

Recall the definition of the energy in (1.10). A change of variable yields,

$$
\mathcal{E}\left(u ; Q_{\ell, j}\right) \geq \frac{1}{L^{2 / 3}\left|a_{j, 2}\right|} e_{N}\left((1-\eta)\left|a_{j, 2}\right| L^{2 / 3}, \sqrt{\left|a_{j, 2}\right|} \ell\right)-C \eta^{-1} \ell^{4} \int_{Q_{\ell, j}}|u|^{2} d x .
$$

Let us introduce the two new sets of indices,

$$
\begin{aligned}
& \tilde{\mathcal{J}}=\left\{j \in \mathcal{J}: Q_{\ell, j} \cap\left\{\left|x_{2}\right| \leq \frac{1}{2} b_{0}(1-\eta)^{-1} L^{-2 / 3}\right\} \neq \emptyset\right\} \\
& \quad \text { and } \mathcal{J}_{\infty}=\left\{j \in \mathcal{J}: Q_{\ell, j} \subset\left\{\left|x_{2}\right| \geq \frac{1}{2} b_{0}(1-\eta)^{-1} L^{-2 / 3}\right\}\right\} .
\end{aligned}
$$

Note that $\mathcal{J}=\tilde{\mathcal{J}} \cup \mathcal{J}_{\infty}$ and we can decompose every sum over $\mathcal{J}$ in the following obvious way 


$$
\sum_{j \in \mathcal{J}}=\sum_{j \in \widetilde{\mathcal{J}}}+\sum_{j \in \mathcal{J}_{\infty}}
$$

Furthermore, the set $\widetilde{\mathcal{J}}$ is non-empty if $A<\frac{1}{2} b_{0}(1-\eta)^{-1} L^{-2 / 3}$. Since $\eta \in\left(0, \frac{1}{2}\right)$ and $b_{0} \geq 2$, this last condition is satisfied when $0<L \leq A^{-3 / 2}$. We will assume this condition henceforth.

Since $\left|a_{j, 2}\right| \geq A$ and $b_{0} \geq 1$, then the condition in (3.5) ensures that

$$
\sqrt{\left|a_{j, 2}\right|} \ell \geq 2 \sqrt{b_{0}}>1 \text {. }
$$

Now, if $j \in \mathcal{J}$, then we can use the lower bound in Remark 2.4 with $b=(1-\eta)\left|a_{j, 2}\right| L^{2 / 3}$ and $r=\sqrt{\left|a_{j, 2}\right|} \ell$ to write, for a different constant $C>0$,

$$
\mathcal{E}\left(u ; Q_{\ell, j}\right) \geq L^{-2 / 3} \ell^{2}\left(g\left((1-\eta)\left|a_{j, 2}\right| L^{2 / 3}\right)-\frac{C}{\ell} \sqrt{1-\eta} L^{1 / 3}\right)-C \eta^{-1} \ell^{4} \int_{Q_{\ell, j}}|u|^{2} d x .
$$

If $j \in \mathcal{J}_{\infty}$, then $(1-\eta)\left|a_{j, 2}\right| L^{2 / 3} \geq \frac{1}{2} b_{0}$ and we can use the identity in (3.4) to write

$$
e_{N}\left((1-\eta)\left|a_{j, 2}\right| L^{2 / 3}, \sqrt{\left|a_{j, 2}\right|} \ell\right)=0 .
$$

Now we can infer from (3.9) the following estimate,

$$
\mathcal{E}\left(u ; \mathcal{S}_{R} \cap\left\{x_{2} \geq A\right\}\right) \geq L^{-2 / 3} \sum_{j \in \widetilde{\mathcal{J}}}\left(g\left((1-\eta)\left|a_{j, 2}\right| L^{2 / 3}\right)-\frac{C}{\ell} \sqrt{1-\eta} L^{1 / 3}\right) \ell^{2}-C \eta^{-1} \ell^{4} \int_{\mathcal{S}_{R}}|u|^{2} d x .
$$

Using the assumption on the $L^{2}$-norm of $u$ (see Lemma 3.2), we get further,

$$
\mathcal{E}\left(u ; \mathcal{S}_{R} \cap\left\{x_{2} \geq A\right\}\right) \geq L^{-2 / 3} \sum_{j \in \widetilde{\mathcal{J}}}\left(g\left((1-\eta)\left|a_{j, 2}\right| L^{2 / 3}\right)-\frac{C}{\ell} \sqrt{1-\eta} L^{1 / 3}\right) \ell^{2}-C \eta^{-1} \ell^{4} R L^{-2 / 3} .
$$

For any $j \in \mathcal{J}$, we choose in $\overline{Q_{j, \ell}}$ the previously free point $a_{j}$ as $a_{j}:=\left(c_{j, 1}, c_{j, 2}+\frac{\ell}{2}\right)$.

Since $g(\cdot)$ is a non decreasing function, this choice yields that,

$$
g\left((1-\eta) a_{j, 2} L^{\frac{2}{3}}\right)=\sup _{t \in\left(-\frac{\ell}{2}+c_{j, 2}, c_{j, 2}+\frac{\ell}{2}\right)} g\left((1-\eta) t L^{\frac{2}{3}}\right) .
$$

In that way, the sum

$$
\ell^{2} \sum_{j \in \widetilde{\mathcal{J}}} g\left((1-\eta)\left|a_{j, 2}\right| L^{2 / 3}\right)
$$

is an upper Riemann sum of the function $\left(x_{1}, x_{2}\right) \mapsto g\left((1-\eta)\left|x_{2}\right| L^{\frac{2}{3}}\right)$ on $\mathcal{D}_{L, R}:=\bigcup_{j \in \tilde{\mathcal{J}}} Q_{\ell, j}$ and

$$
\begin{aligned}
\mathcal{E}\left(u ; \mathcal{S}_{R} \cap\left\{x_{2} \geq A\right\}\right) \geq & L^{-2 / 3} \int_{\mathcal{D}_{L, R}} g\left((1-\eta)\left|x_{2}\right| L^{2 / 3}\right) d x_{1} d x_{2}-C(1-\eta)^{-1 / 2} L^{-1} R \\
& -C \eta^{-1} \ell^{4} R L^{-2 / 3} .
\end{aligned}
$$

We now observe that, by definition of $\tilde{\mathcal{J}}$ and $\mathcal{J}$,

$$
\mathcal{D}_{L, R}=\bigcup_{j \in \widetilde{\mathcal{J}}} Q_{\ell, j} \subset\left\{\left(x_{1}, x_{2}\right) \in \mathbb{R}^{2}:\left|x_{1}\right| \leq R / 2 \quad \text { and } \quad A<x_{2} \leq b_{0}(1-\eta)^{-1} L^{-2 / 3}+\ell\right\} .
$$

Since $g(\cdot)$ is valued in $]-\infty, 0]$ and $g(b)=0$ for all $b \geq 1$, then

$$
\int_{\mathcal{D}_{L, R}} g\left((1-\eta)\left|x_{2}\right| L^{2 / 3}\right) d x_{1} d x_{2} \geq \int_{0 \leq x_{2} \leq b_{0}(1-\eta)^{-1} L^{-2 / 3}+\ell}\left(\int_{\left|x_{1}\right| \leq R / 2} g\left((1-\eta)\left|x_{2}\right| L^{2 / 3}\right) d x_{1}\right) d x_{2},
$$


and a simple change of variable yields,

$$
\int_{\mathcal{D}_{L, R}} g\left((1-\eta)\left|x_{2}\right| L^{2 / 3}\right) d x_{1} d x_{2} \geq R(1-\eta)^{-1} L^{-2 / 3} \int_{0}^{1} g(t) d t .
$$

Therefore, we have proved the following lower bound,

$$
\mathcal{E}\left(u ; \mathcal{S}_{R} \cap\left\{x_{2} \geq A\right\}\right) \geq L^{-4 / 3} R(1-\eta)^{-1} \int_{0}^{1} g(t) d t-C(1-\eta)^{-1 / 2} L^{-1} R-C \eta^{-1} \ell^{4} R L^{-2 / 3} .
$$

Now, we choose $n=[R+1]$ where [ $\cdot]$ denotes the integer part. In that way, the condition in (3.5) is satisfied for all $R \geq 1$ and $A \geq A_{0}=4 \sqrt{b_{0}}$. Moreover, we have the lower bound,

$$
\mathcal{E}\left(u ; \mathcal{S}_{R} \cap\left\{x_{2} \geq A\right\}\right) \geq 2 L^{-4 / 3} R(1-\eta)^{-1} \int_{0}^{1} g(t) d t-C(1-\eta)^{-1 / 2} L^{-1} R-C \eta^{-1} R L^{-2 / 3} .
$$

Now, we choose $\eta=\frac{1}{2} L^{1 / 3}$ so that, for all $L \in(0,1), \eta \in\left(0, \frac{1}{2}\right), \eta^{-1} L^{-2 / 3}=2 L^{-1}, \eta L^{-4 / 3}=\frac{1}{2} L^{-1}$ and the lower bound in (3.2) is satisfied.

Proof of Theorem 3.1. We use the conclusion in Lemma 3.2 with the following choices,

$$
R=4, \quad A=A_{0}, \quad 0<L \leq L_{0}:=A^{-3 / 2}, \quad u=\varphi_{L, R},
$$

where $\varphi_{L, R}$ is a minimizer of $\mathcal{E}_{L, R}$. Notice that, the estimates in Proposition 2.6 ensure that the function $u=\varphi_{L, R}$ satisfies the assumptions in Lemma 3.2.

Thanks to (2.6), we may write,

$$
E(L) \geq \frac{\mathcal{E}_{L, R}\left(\varphi_{L, R}\right)}{R}-C\left(1+L^{-2 / 3}\right) .
$$

By splitting the integral over $\mathcal{S}_{R}$ into two parts

$$
\int_{\mathcal{S}_{R}}=\int_{\mathcal{S}_{R} \cap\left\{\left|x_{2}\right| \geq A\right\}}+\int_{\mathcal{S}_{R} \cap\left\{\left|x_{2}\right| \leq A\right\}}
$$

then using that

$$
\left|\left(\nabla-i \mathbf{A}_{\mathrm{van}}\right) \varphi_{L, R}\right|^{2}-L^{-2 / 3}\left|\varphi_{L, R}\right|^{2}+\frac{L^{-2 / 3}}{2}\left|\varphi_{L, R}\right|^{4} \geq-L^{-2 / 3}\left|\varphi_{L, R}\right|^{2},
$$

we get,

$$
\begin{aligned}
\mathcal{E}_{L, R}\left(\varphi_{L, R}\right) \geq & \int_{\mathcal{S}_{R} \cap\left\{\left|x_{2}\right| \geq A\right\}}\left(\left|\left(\nabla-i \mathbf{A}_{\mathrm{van}}\right) \varphi_{L, R}\right|^{2}-L^{-2 / 3}\left|\varphi_{L, R}\right|^{2}+\frac{L^{-2 / 3}}{2}\left|\varphi_{L, R}\right|^{4}\right) d x \\
& -\int_{\mathcal{S}_{R} \cap\left\{\left|x_{2}\right| \leq A\right\}} L^{-2 / 3}\left|\varphi_{L, R}\right|^{2} d x .
\end{aligned}
$$

Now, we use the conclusion in Lemma 3.2 and the bound $\left\|\varphi_{L, R}\right\|_{\infty} \leq 1$ to write,

$$
\mathcal{E}_{L, R}\left(\varphi_{L, R}\right) \geq 2 R L^{-4 / 3} \int_{0}^{1} g(b) d b-C R L^{-1}-2 A R L^{-2 / 3} .
$$

We insert this into (3.12) to finish the proof of Theorem 3.1. 


\section{Proof of Theorem 1.2: upper bound}

The aim of this section is to prove the following upper bound version of Theorem 1.2. Note that we provide an explicit control of the remainder term.

Theorem 4.1. There exist two constants $L_{0}>0$ and $C>0$ such that, for all $L \in\left(0, L_{0}\right)$,

$$
E(L) \leq 2 L^{-4 / 3} \int_{0}^{1} g(b) d b+C L^{-1 / 3},
$$

where $E(\cdot)$ and $g(\cdot)$ are the energies introduced in (1.18) and (1.11) respectively.

The proof of Theorem 4.1 relies on the following lemma:

Lemma 4.2. Let $R \geq 1, L>0, \ell \in(0,1), \eta \in(0,1), c=\left(c_{1}, c_{2}\right) \in \mathbb{R}^{2}$ and

$$
Q_{\ell}=\left(-\ell / 2+c_{1}, c_{1}+\ell / 2\right) \times\left(-\ell / 2+c_{2}, c_{2}+\ell / 2\right) .
$$

Suppose that

$$
Q_{\ell} \subset\left\{\left(x_{1}, x_{2}\right) \in \mathbb{R}^{2}:\left|x_{1}\right| \leq R / 2 \text { and }\left|x_{2}\right| \geq \frac{1}{\ell^{2}}\right\} .
$$

For all $R \geq 1$, it holds,

$$
\begin{aligned}
& \inf \left\{\mathcal{E}_{L, R}(w): w \in H_{0}^{1}\left(Q_{\ell}\right)\right\} \\
& \quad \leq L^{-2 / 3} \int_{Q_{\ell}} g\left((1+\eta) L^{2 / 3}\left|x_{2}\right|\right) d x_{1} d x_{2}+C L^{-2 / 3}\left(\ell^{-1} L^{1 / 3}+\eta^{-1} \ell^{4}\right) \ell^{2},
\end{aligned}
$$

where, for all $w \in H_{0}^{1}\left(Q_{\ell}\right), \mathcal{E}_{L, R}(w)$ is introduced in (1.15) by setting $w=0$ outside $Q_{\ell}$, and $C>0$ is a constant independent of $\ell, \eta, c, L$ and $R$.

Proof. We write the details of the proof when $Q_{\ell} \subset\left\{x_{2} \geq \ell^{-2}\right\}$. The case $Q_{\ell} \subset\left\{x_{2} \leq-\ell^{-2}\right\}$ can be handled similarly. Let $a=\left(a_{1}, a_{2}\right) \in \overline{Q_{\ell}}$. As we did in the derivation of (3.7), we may define a smooth function $\phi$ in $Q_{\ell}$ such that,

$$
\mathbf{A}_{\operatorname{van}}(x)=a_{2} \mathbf{A}_{0}(x-c)+\mathbf{F}(x)-\nabla \phi(x) \text { in } Q_{\ell},
$$

and

$$
|\mathbf{F}(x)| \leq C \ell^{2} \quad \text { in } Q_{\ell},
$$

where $C>0$ is a universal constant.

We introduce the following three parameters,

$$
\eta \in(0,1), \quad b=a_{2}(1+\eta) L^{2 / 3}, \quad r=\sqrt{a_{2}} \ell .
$$

Define the following function,

$$
u(x)=e^{i \phi(x)} u_{b, r}\left(\sqrt{a_{2}}(x-c)\right), \quad x \in Q_{\ell},
$$

where $u_{b, r} \in H_{0}^{1}\left(Q_{r}\right)$ is a minimizer of the energy $e_{D}(b, r)$ in (1.9).

Clearly, $u \in H_{0}^{1}\left(Q_{\ell}\right)$. Hence,

$$
\inf \left\{\mathcal{E}_{L, R}(w): w \in H_{0}^{1}\left(Q_{\ell}\right)\right\} \leq \mathcal{E}_{L, R}(u) .
$$

Using (4.1) and the Cauchy-Schwarz inequality, we compute the energy of $u$ as follows, 


$$
\begin{aligned}
\mathcal{E}_{L, R}(u) \leq & \int_{Q_{\ell}}\left((1+\eta)\left|\left(\nabla-i a_{2} \mathbf{A}_{0}(x-c)\right) e^{-i \phi} u\right|^{2}-L^{-2 / 3}|u|^{2}+\frac{L^{-2 / 3}}{2}|u|^{4}\right) d x \\
& +4 \eta^{-1} \int_{Q_{\ell}}|\mathbf{F}(x)|^{2}|u|^{2} d x .
\end{aligned}
$$

Using (4.2), the bound $\left|u_{b, r}\right| \leq 1$, a change of variable and (4.3), we get,

$$
\mathcal{E}_{L, R}(u) \leq \frac{L^{-2 / 3}}{a_{2}} F_{b, r}\left(u_{b, r}\right)+C \eta^{-1} \ell^{6},
$$

where $F_{b, r}$ is the functional in (1.7).

Our choice of $u_{b, r}$ ensures that,

$$
F_{b, r}\left(u_{b, r}\right)=e_{D}(b, r) .
$$

Again, thanks to the choice of $b$ and $r$ in (4.3), we get,

$$
\mathcal{E}_{L, R}(u) \leq \frac{L^{-2 / 3}}{a_{2}} e_{D}\left((1+\eta) a_{2} L^{2 / 3}, \sqrt{a_{2}} \ell\right)+C \eta^{-1} \ell^{6} .
$$

Now, by the assumption $Q_{\ell} \subset\left\{x_{2} \geq \ell^{-2}\right\}$, we know that $\sqrt{a_{2}} \ell \geq 1$. Thus we may use (2.2) to write,

$$
\begin{aligned}
\mathcal{E}_{L, R}(u) & \leq \frac{L^{-2 / 3}}{a_{2}}\left(g\left((1+\eta) a_{2} L^{2 / 3}\right)+\frac{C \sqrt{1+\eta} L^{1 / 3}}{\ell}\right)\left(\sqrt{a_{2}} \ell\right)^{2}+C \eta^{-1} \ell^{6} \\
& =L^{-2 / 3}\left(g\left((1+\eta) a_{2} L^{2 / 3}\right)+\frac{C \sqrt{1+\eta} L^{1 / 3}}{\ell}\right) \ell^{2}+C \eta^{-1} \ell^{6},
\end{aligned}
$$

which is uniformly true for $a \in \overline{Q_{\ell}}$.

We now select $a=\left(c_{1}, c_{2}-\frac{\ell}{2}\right)$. Since $g(\cdot)$ is a non-decreasing function, then

$$
g\left((1+\eta) a_{2} L^{2 / 3}\right)=\inf _{x_{2} \in\left(-\frac{\ell}{2}+c_{2}, c_{2}+\frac{\ell}{2}\right)} g\left((1+\eta) x_{2} L^{2 / 3}\right) .
$$

This yields,

$$
\ell^{2} g\left((1+\eta) a_{2} L^{2 / 3}\right) \leq \int_{Q_{\ell}} g\left((1+\eta) x_{2} L^{2 / 3}\right) d x_{1} d x_{2},
$$

and finishes the proof of Lemma 4.2.

Proof of Theorem 4.1. Let $R=4, L \in(0,1), \eta=L$ and $\ell=\frac{1}{4}$. Let $\left(Q_{\ell, j}\right)_{j}$ be the lattice of squares generated by the square

$$
Q=(-R / 2,-R / 2+\ell) \times\left(\ell^{-2}, \ell^{-2}+\ell\right) .
$$

Define the set of indices

$$
\mathcal{J}=\left\{j: Q_{\ell, j} \subset \mathcal{S}_{R} \cap\left\{x_{2} \geq \ell^{-2}\right\} \quad \text { and } Q_{\ell, j} \cap\left\{x_{2} \leq(1+\eta)^{-1} L^{-2 / 3}\right\} \neq \emptyset\right\} .
$$

For all $x=\left(x_{1}, x_{2}\right) \in \mathbb{R}^{2}$ with $x_{2} \geq 0$, define $u(x)$ as follows,

$$
u(x)= \begin{cases}u_{\ell, j}(x) & \text { if } j \in \mathcal{J}, \\ 0 & \text { if } j \notin \mathcal{J},\end{cases}
$$

where $u_{\ell, j} \in H_{0}^{1}\left(Q_{\ell, j}\right)$ is a minimizer of the following ground state energy

$$
\inf \left\{\mathcal{E}_{L, R}(w): w \in H_{0}^{1}\left(Q_{\ell, j}\right)\right\} .
$$

We extend $u(x)$ in $\left\{x_{2} \leq 0\right\}$ as follows, 


$$
u(x)=\bar{u}\left(x_{1},-x_{2}\right), \quad x=\left(x_{1}, x_{2}\right) \quad \text { and } x_{2} \leq 0 .
$$

Clearly, $u \in H_{\mathrm{mag}, 0}^{1}\left(\mathcal{S}_{R}\right)$. Notice that,

$$
\mathcal{E}_{L, R}(u)=2 \sum_{j \in \mathcal{J}} \mathcal{E}_{L, R}\left(u_{\ell, j}\right),
$$

and for $j \in \mathcal{J}$, the square $Q_{\ell, j}$ satisfies the assumption in Lemma 4.2. We use Lemma 4.2 to write,

$$
\mathcal{E}_{L, R}(u) \leq 2 L^{-2 / 3} \int_{\mathcal{D}_{\ell}} g\left((1+\eta) L^{2 / 3} x_{2}\right) d x_{1} d x_{2}+C L^{-1 / 3}\left|\mathcal{D}_{\ell}\right|,
$$

where the domain $\mathcal{D}_{\ell}$ is given as follows,

$$
\mathcal{D}_{\ell}=\bigcup_{j \in \mathcal{J}} \overline{Q_{\ell, j}} .
$$

Thanks to the definition of the set $\mathcal{J}$, it is clear that,

$$
\mathcal{S}_{R} \cap\left\{\ell^{-2} \leq x_{2} \leq(1+\eta)^{-1} L^{-2 / 3}\right\} \subset \mathcal{D}_{\ell} \subset \mathcal{S}_{R} \cap\left\{0 \leq x_{2} \leq(1+\eta)^{-1} L^{-2 / 3}+\ell\right\} .
$$

This yields:

$$
\left|\mathcal{D}_{\ell}\right|=\mathcal{O}\left(R L^{-1 / 3}\right)
$$

and (since the function $g(\cdot)$ is valued in $\left[-\frac{1}{2}, 0\right]$ and $g(b)=0$ for all $b \geq 1$ ),

$$
\begin{aligned}
\int_{\mathcal{D}_{\ell}} g\left((1+\eta) L^{2 / 3} x_{2}\right) d x_{1} d x_{2} & \leq \int_{\ell^{-2}}^{(1+\eta)^{-1}} \int_{-R / 2}^{L^{-2 / 3}} g\left((1+\eta) L^{2 / 3} x_{2}\right) d x_{1} d x_{2} \\
& =(1+\eta)^{-1} L^{-2 / 3} R \int_{\ell^{-2}(1+\eta) L^{2 / 3}}^{1} g(t) d t \\
& \leq(1+\eta)^{-1} L^{-2 / 3} R \int_{0}^{1} g(t) d t+\ell^{-2} R .
\end{aligned}
$$

Substitution into (4.4) yields (recall that $\eta=L \in(0,1)$ and $\ell=\frac{1}{4}$ ),

$$
\mathcal{E}_{L, R}(u) \leq 2 L^{-4 / 3} R \int_{0}^{1} g(t) d t+C R L^{-1 / 3} .
$$

Since $u \in H_{\mathrm{mag}, 0}^{1}\left(\mathcal{S}_{R}\right)$, then

$$
\mathfrak{e}_{\mathrm{gs}} \leq \mathcal{E}_{L, R}(u) \leq 2 L^{-4 / 3} R \int_{0}^{1} g(t) d t+C R L^{-1 / 3} .
$$

We divide by $R$ and use (2.5) to deduce that

$$
E(L) \leq 2 L^{-4 / 3} R \int_{0}^{1} g(t) d t+C L^{-1 / 3} .
$$




\section{Proof of Theorem 1.3}

Let $\ell \in(0,1)$ be a parameter independent of $\kappa$. Define the two sets,

$$
\Omega_{\kappa, \ell}=\left\{x \in \Omega:\left|B_{0}(x)\right|<\frac{1}{b(\kappa) \kappa} \quad \text { and } \operatorname{dist}(x, \partial \Omega)>\ell\right\}, \quad \Gamma_{\kappa, \ell}=\{x \in \Gamma: \operatorname{dist}(x, \partial \Omega)>\ell\} .
$$

Recall that $\Gamma=\left\{B_{0}=0\right\}$ and by Assumption 1.1, $\Gamma \cap \partial \Omega$ is a finite set. Thus, the area of $\Omega_{\kappa, \ell}$ and the length of $\Gamma_{\kappa, \ell}$ satisfy, for $\kappa$ sufficiently large and some constant $C>0$ (independent of $\kappa$ and $\ell$ ),

$$
\left|\Omega_{\kappa, \ell}\right| \leq \frac{C \varepsilon(\ell)}{b(\kappa) \kappa}, \quad\left|\Gamma_{\kappa, \ell}\right| \leq C \varepsilon(\ell),
$$

where $\varepsilon(\cdot)$ is a function independent of $\kappa$ and satisfying $\lim _{\ell \rightarrow 0_{+}} \varepsilon(\ell)=0$.

The standard proof of (5.1) is left to the reader. The estimate in (5.1) is easier to verify under the additional assumption that $\Gamma$ and $\partial \Omega$ intersect transversally, and in this case $\varepsilon(\ell)=\ell$. Note that $g(\cdot)$ vanishes in $[1, \infty)$. Thus,

$$
\int_{\Omega} g\left(b(\kappa) \kappa\left|B_{0}(x)\right|\right) d x=\int_{\Omega_{\kappa, \ell}} g\left(b(\kappa) \kappa\left|B_{0}(x)\right|\right) d x+\mathcal{O}\left(\frac{\varepsilon(\ell)}{b(\kappa) \kappa}\right) .
$$

Since $b(\kappa) \kappa \rightarrow+\infty$, then Assumption 1.1 yields, for $\kappa$ sufficiently large,

$$
\exists C>0, \quad \forall x \in \Omega_{\kappa},\left.\quad|| \nabla B_{0}(x)\right|^{-1}-\left|\nabla B_{0}(p(x))\right|^{-1} \mid \leq \frac{C}{b(\kappa) \kappa} .
$$

Here, for $\kappa$ sufficiently large and for all $x \in \Omega_{\kappa, \ell}$, the point $p(x) \in \Gamma$ is uniquely defined by the relation

$$
\operatorname{dist}(x, \Gamma)=\operatorname{dist}(x, p(x)) .
$$

The co-area formula yields,

$$
\int_{\Omega_{\kappa, \ell}} g\left(b(\kappa) \kappa\left|B_{0}(x)\right|\right) d x=\int_{0}^{\frac{1}{b(\kappa) \kappa}}\left(\int_{\left\{\left|B_{0}\right|=r\right\} \cap \Omega_{\kappa, \ell}}\left|\nabla B_{0}(x)\right|^{-1} g(b(\kappa) \kappa r) d s\right) d r .
$$

Thanks to (5.3), we get further,

$$
\begin{aligned}
& \int_{\Omega_{\kappa, \ell}} g\left(b(\kappa) \kappa\left|B_{0}(x)\right|\right) d x \\
& =\int_{0}^{\frac{1}{b(\kappa) \kappa}}\left(\int_{\left\{\left|B_{0}\right|=r\right\} \cap \Omega_{\kappa, \ell}}\left|\nabla B_{0}(p(x))\right|^{-1} g(b(\kappa) \kappa r) d s\right) d r+\mathcal{O}\left(\frac{1}{(b(\kappa) \kappa)^{2}}\right) .
\end{aligned}
$$

Now, a simple calculation yields,

$$
\begin{aligned}
& \int_{0}^{\frac{1}{b(\kappa) \kappa}}\left(\int_{\left\{\left|B_{0}\right|=r\right\} \cap \Omega_{\kappa, \ell}}\left|\nabla B_{0}(p(x))\right|^{-1} g(b(\kappa) \kappa r) d s\right) d r \\
& =\int_{0}^{\frac{1}{b(\kappa) \kappa}}\left(\int_{\left\{\left|B_{0}\right|=r\right\} \cap \Omega_{\kappa, \ell}}\left|\nabla B_{0}(p(x))\right|^{-1} d s\right) g(b(\kappa) \kappa r) d r,
\end{aligned}
$$

and (using a simple analysis of the arc-length measure in the curve $\left\{\left|B_{0}\right|=r\right\}$ and the assumption that $\Gamma \cap \partial \Omega$ is a finite set), 


$$
\begin{aligned}
\forall r \in\left(0, \frac{1}{b(\kappa) \kappa}\right), \quad & \int_{\left\{\left|B_{0}\right|=r\right\} \cap \Omega_{\kappa, \ell}}\left|\nabla B_{0}(p(x))\right|^{-1} d s \\
= & \int_{\left\{\left|B_{0}\right|=0\right\}}\left|\nabla B_{0}(p(x))\right|^{-1} d s+\mathcal{O}(\eta(\kappa)+\varepsilon(\ell)), \quad(\kappa \rightarrow \infty),
\end{aligned}
$$

where $\eta(\cdot)$ satisfies

$$
\lim _{\kappa \rightarrow \infty} \eta(\kappa)=0 .
$$

As a consequence, we get the following formula,

$$
\int_{\Omega_{\kappa, \ell}} g\left(b(\kappa) \kappa\left|B_{0}(x)\right|\right) d x=\int_{\Gamma}\left(\int_{0}^{\frac{1}{b(\kappa) \kappa}} g(b(\kappa) \kappa r) d r\right)\left|\nabla B_{0}(x)\right|^{-1} d s(x)+\mathcal{O}\left(\frac{\eta(\kappa)+\varepsilon(\ell)}{b(\kappa) \kappa}\right)
$$

A change of variable and Theorem 1.2 yield,

$$
\begin{aligned}
\int_{0}^{\frac{1}{b(\kappa) \kappa}} g(b(\kappa) \kappa r) d r & =\frac{1}{b(\kappa) \kappa} \int_{0}^{1} g(t) d t \\
& =\frac{1}{2 b(\kappa) \kappa}\left(L^{4 / 3} E(L)+\varepsilon_{1}(L)\right),
\end{aligned}
$$

where $\lim _{L \rightarrow 0} \varepsilon_{1}(L)=0$.

For $\kappa$ sufficiently large, we take

$$
L=b(\kappa)\left|\nabla B_{0}(x)\right|,
$$

and get,

$$
\int_{\Omega_{\kappa, \ell}} g\left(b(\kappa) \kappa\left|B_{0}(x)\right|\right) d x=\frac{1}{2 \kappa} \int_{\Gamma}\left|\nabla B_{0}(x)\right|^{1 / 3} E\left(b(\kappa)\left|\nabla B_{0}(x)\right|\right) d s(x)+\mathcal{O}\left(\frac{\lambda(\kappa)+\eta(\kappa)+\varepsilon(\ell)}{b(\kappa) \kappa}\right),
$$

where $\lambda(\cdot)$ satisfies $\lim _{\kappa \rightarrow \infty} \lambda(\kappa)=0$. Inserting this into (5.2) and noticing that $\lambda(\kappa), \eta(\kappa) \rightarrow 0$ as $\kappa \rightarrow \infty, \varepsilon(s) \rightarrow 0$ as $s \rightarrow 0_{+}$and $\ell$ was arbitrary in $(0,1)$, then we get the conclusion in Theorem 1.3.

\section{Conflict of interest statement}

The authors declare there is no conflict of interest.

\section{Acknowledgements}

This work was done when the first author was Simons foundation Visiting Fellow at the Isaac Newton Institute in Cambridge. The support of the ANR project Nosevol (No. 2011BS0101901) is also acknowledged. The second author acknowledges financial support through a fund from Lebanese University.

\section{References}

[1] K. Attar, The ground state energy of the two dimensional Ginzburg-Landau functional with variable magnetic field, Ann. Inst. Henri Poincaré, Anal. Non Linéaire. 32 (2015) 325-345.

[2] K. Attar, Energy and vorticity of the Ginzburg-Landau model with variable magnetic field, Asymptot. Anal. 93 (2015) $75-114$.

[3] K. Attar, Pinning with a variable magnetic field of the two dimensional Ginzburg-Landau model, preprint, arXiv:1503.06500, 2015.

[4] V. Bonnaillie, On the fundamental state for a Schrödinger operator with magnetic fields in domains with corners, Asymptot. Anal. 41 (3-4) (2015) 215-258. 
[5] A. Contreras, X. Lamy, Persistence of superconductivity in thin shells beyond $H_{c 1}$, arXiv:1411.1078v1 [math.AP], 2014, Commun. Contemp. Math., http://dx.doi.org/10.1142/S0219199715500479.

[6] S. Fournais, B. Helffer, Spectral Methods in Surface Superconductivity, Prog. Nonlinear Differ. Equ. Appl., vol. 77, Birkhäuser, 2010.

[7] S. Fournais, A. Kachmar, The ground state energy of the three dimensional Ginzburg-Landau functional. Part I. Bulk regime, Commun. Partial Differ. Equ. 38 (2013) 339-383.

[8] P.G. de Gennes, Boundary effects in superconductors, Rev. Mod. Phys. (January 1964).

[9] B. Helffer, A. Kachmar, The Ginzburg-Landau functional with a vanishing magnetic field, Arch. Ration. Mech. Anal. 218 (2015) 55-122, http://dx.doi.org/10.1007/s00205-015-0856-z.

[10] R. Montgomery, Hearing the zero locus of a magnetic field, Commun. Math. Phys. 168 (3) (1995) 651-675.

[11] J.-P. Miqueu, Equation de Schrödinger avec un champ magnétique qui s'annule, Thèse de doctorat, in preparation.

[12] X.B. Pan, Upper critical field for domains with edges and corners, Calc. Var. Partial Differ. Equ. 14 (4) (2002) $447-482$.

[13] X.B. Pan, K.H. Kwek, Schrödinger operators with non-degenerately vanishing magnetic fields in bounded domains, Trans. Am. Math. Soc. 354 (10) (2002) 4201-4227.

[14] E. Sandier, S. Serfaty, Vortices for the Magnetic Ginzburg-Landau Model, Progress in Nonlinear Differential Equations and their Applications, vol. 70, Birkhäuser, 2007.

[15] E. Sandier, S. Serfaty, The decrease of bulk superconductivity close to the second critical field in the Ginzburg-Landau model, SIAM J. Math. Anal. 34 (4) (2003) 939-956. 